\title{
Pluripotent stem cells and neurological diseases
}

ALYSSON RENATO MUOTRI

\section{Introduction}

$\mathrm{B}$ IOPSIES OF the peripheral nervous system are frequent and often performed with the aim to investigate the disease process in some patients. However, due to the extremely invasive nature of the procedure, biopsies of the central nervous system (CNS) are performed only in special and rare circumstances. This inability to explore the brain of a living individual limits considerably our knowledge about the advance of neurodevelopmental and neurodegenerative diseases. Most of our current knowledge about cellular phenotypes related to CNS diseases in humans was gathered from studies performed in brain tissue collected postmortem, which is not necessarily preserved appropriately. Moreover, in most cases the tissues represent only the end-stage of the disease, eliminating the possibility of exploring the initial events responsible for the cascade of cellular changes that lead to the final result, be it structural changes or even cell death.

Animal models are extremely useful because they can reproduce various forms of human genetic neurodegenerative diseases. Transgenic models or knockouts have produced numerous insights into new molecular mechanisms behind many human diseases, creating new possibilities for therapeutic intervention. Currently, these models are restricted to monogenetic diseases, limiting the use range and representing only a small number of human diseases that can be modeled. Technical difficulties, interspecies differences and differences in genetic backgrounds end up interfering in the animal model process, even in the case of monogenetic diseases. In many cases, transgenic or knockout technology is incapable of reproducing human symptoms in animals, indicating a clear need for human models.

The original cell reprogramming experiments led by Japanese researcher Shinya Yamanaka surprised the scientific community by breaking the dogma that specialized cells in the human body would have a lifetime identity (Takahashi et al., 2007). The forced expression of a group of transcription factors, genes related to the pluripotent state, has the ability to redirect the identity of specialized cells and represents a superb way to demonstrate cellular flexibility. This induced return to the pluripotent embryonic stage was dubbed iPSC (Induced Pluripotent Stem Cells). 
Concisely, gene reprogramming involves the return to a more potent and plastic form from an already differentiated or specialized cell. For example, the skin cell of an adult can be transformed into an unspecialized, undifferentiated cell, capable of dividing indefinitely. This undifferentiated and immortal cell would have the potential to specialize again in the same skin cell or in any other cell type, including a neuron. In summary, it is possible to obtain embryoniclike stem cells on demand, using the same genetic material of the patient and avoiding possible rejection in case of transplants.

Although the idea of a transplant without rejection is extremely attractive to those interested in a possible cell therapy, Yamanaka's experiments enable turning into reality the dream of many neuroscientists: to capture the human genome of a patient in pluripotent stem cells and use them for unlimited production of specialized cells of the nervous system (Muotri, 2009). Although iPSC generated from the patient are, in theory, less immunogenic for possible transplant therapies, the focus of this article is geared toward future clinical applications of this technology in vitro, such as modeling neurological diseases and using biotechnology for the discovery of novel drugs through drug screening.

\section{iPSC generated from human neurodegenerative diseases}

Diseases that can be modeled by cellular reprogramming may be rare, monogenetic or in the broad spectrum of sporadic and multifactorial diseases. To date, there is no scientific publication demonstrating the usefulness of iPSC technology to model the latter group of complex diseases. The difficulty to obtain conclusive results from this type of complex illness is possibly due to different genetic backgrounds and environmental/epigenetic influences. It is worth noting that even monogenetic diseases can show a large phenotypic variability. It will be necessary to determine whether the genotype-phenotype variation observed in patients will be reproduced by neurons generated from the patients' iPSC or if reprogramming will eliminate the epigenetic or environmental "noise".

Reprogramming of somatic cells has been published for some neurological diseases. However, a critical reading reveals that few studies have actually shown that it is possible to recapitulate the human phenotype in iPSC-derived neurons. The derivation of neurons from iPSC has been described for sporadic forms of neurodegenerative diseases with late symptoms such as amyotrophic lateral sclerosis (ALS) and Parkinson's disease (Park et al. 2008; Soldner et al. 2009; Dimos et al. 2008). From the reprogramming point of view, it is remarkable that aged fibroblasts (up to 85 years old) from ALS and PD patients could still be reset with similar efficiency as fibroblasts from younger patients. Despite the excitement generated by these early studies, none of them showed phenotypes related to the disease in affected cells. It is possible that cellular phenotypes will arise only over the years, precisely as it happens in the patients' bodies. This would make the process highly ineffective due to the technical difficulties of maintaining functional neurons in culture for such a long time. Identifying 
situations that mimic premature aging, for example, by culturing cells under increased oxidative stress seems to be a creative solution.

Partial modeling with the spontaneous phenotype generation in the laboratory was observed for two monogenetic diseases with early symptoms: spinal muscular atrophy (SMA) and Familial dysautonomia (FD) (Ebert et al., 2009; Lee et al., 2009). Both are autosomal recessive diseases, which have in common the rapid progression in the early years of life. In addition, both diseases are associated with loss of function of genes involved in RNA processing. SMA represents a group of recessive disorders caused by deletions or mutations in SMN (Survival Motor Neuron) genes. The SMN1 gene encodes a protein involved in RNA processing (Lorson et al. 1998). SMA type 1 is characterized by mutations in the SMN1 gene, leading to a rapid degeneration of motor neurons, which results in severe muscle atrophy. The symptoms appear around six months of age and the patient's death occurs by respiratory disability before the age of two. iPSC cells from a single SMA patient have been characterized and differentiated into motor neurons. When compared to iPSC-generated motor neurons from a healthy maternal control (non-carriers of the disease), it was found that the SMA-iPSC generated a small number of motor neurons, suggesting the premature death of this cell type. The study does not provide a functional characterization of the motor neurons generated by the group (ability to stimulate potentials or action or form neuromuscular junctions). The group also observed an increase in the number of protein aggregates known as "gems" in fibroblasts and iPSC from the SMA patient. These gems are often directly associated with disease intensity. The aggregates were converted into fibroblasts or SMA-iPSC with the use of specific drugs. Unfortunately, the group did not analyze the presence of these gems in the cells of interest, i.e., the motor neurons. This study showed for the first time a proof of principle that iPSC can be used as a future platform for screening drugs that can reverse or mitigate cellular phenotypes associated with human diseases. Unfortunately, the initial enthusiasm was cooled by the absence of stringer controls and other SMA patients. Incorporation of additional control, loss and gain of function would have appeased the concern that the observed phenotype would be a consequence of the intrinsic iPSC variability system (discussed next).

FD is also a recessive disease that occurs at increased frequency in individuals of Ashkenazi Jewish descent (Brunt \& McKusick, 1970). The disease is characterized by degeneration of sensory and autonomic neurons, leading to severe and lethal general dysfunction. Clinical features include alacrima, hypoactivity and relative indifference to pain and temperature. A splicing defect in the IKBKAP gene results in a tissue-specific loss of protein function (Slaugenhaupt et al. 2001). iPSC derived from three FD patients were differentiated into progenitor cells and revealed low levels of IKBKAP expression when compared to the control group. Defects in neuronal differentiation and migration were also observed in iPSC-FD- 
derived neural progenitor cells. A drug (kinetin) was used to partly recover mutant IKBKAP RNA splicing in these cells, reversing the migration defects but not neuronal differentiation defects. Kinetin chemical variations could have been used in an attempt to reverse both defects. Anyway, this second study has advantages over the first, particularly because it includes more than one patient.

\section{Modeling neurodevelopmental disorders}

Although advances in iPSC modeling have been restricted thus far to neurodegenerative diseases, I anticipate that the technology will be useful for modeling neurodevelopmental and psychiatric disorders such as autism and schizophrenia. However, I believe that initial results will probably emerge from monogenetic, early-onset diseases of known causes. An example of a human disease with great modeling potential is Rett syndrome, which is characterized by arrested development in early childhood, regression of acquired skills, loss of speech, stereotypical movements, and a broad range of autistic characteristics (Samaco et al. 2005). Rett female patients are generally heterozygous for mutations in the X-linked MeCP2 gene. MeCP2 protein shows higher affinity for methylated DNA and seems to be involved in epigenetic regulation of target genes in the nervous system. Knowing the gene involved in this syndrome will allow genetic manipulation in iPSC-derived neurons from patients with Rett syndrome, validating any cellular or molecular phenotypes found after differentiation. This important validation will be essential to convince us that the observed phenotypes are not the result of experimental variations.

Rett syndrome is one of the syndromes associated with ASD and perhaps the ideal prototype to start investigating complex psychiatric diseases. Following this model, the next step would be to seek similar phenotypes in several ASD syndromes, seeking common molecular pathways. A major challenge for this category of disorders would lie in distinguishing the contribution of genetic background and environmental factors. New tools for the genetic manipulation of pluripotent stem cells, such as homologous recombination and the use of specific zinc-finger endonucleases, may help to eliminate noise or experimental variability (Zwaka \& Thomson 2003; Hockemeyer et al. 2009). An alternative for revealing complex and neuronal phenotypes and specific neural behaviors could be the use of chimeric animals. Human embryonic stem cells transplanted into the brain of murine embryos revealed the enormous adaptation potential of pluripotent cells as a result of contact with the cell niche (Muotri et al., 2005). This type of modeling puts together the human cellular genetic background and the manipulation of an animal body, thus enabling studying the effects of environmental changes in human neurons.

\section{Using human neurons as a tool for screening new drugs}

In the past, drug candidates were selected using human cell strains and 
represented a great advance in medicine. An example was the development of polio vaccines, originally based developed in vitro, using the HeLa cell strain (Syverton et al., 1954). Patient-derived iPSC can offer a much bigger advantage in relation to the traditional transformed cell line model, as they take into consideration the donor's original genome and have the ability to differentiate the specific cell type affected at the exact time of development.

One of the great benefits of reprogramming cells is the possibility of studying the developmental steps of progenitor neural cells before they completely mature into functional neurons. Neural progenitor cells can give rise to both glial and neuronal populations. These populations can further differentiate into specific subtypes of neurons, for example, generating various cell types responsible for the development and formation of the brain (Muotri \& Gage, 2006). It is very likely that some diseases affect only a specific subtype of neuron that has its origins back in the precursor phase. In these cases, therapeutic interventions should occur at the exact stage of development. In addition to these advantages, iPSC provide the unique opportunity for testing drugs directly in human neural networks, something that was hardly imagined a few years ago.

The observation of cell phenotypes during drug screening can greatly benefit from well-established neuroscience techniques. The analysis of single cells can involve neuronal morphology, branching, polarity, neuronal spine density and maturation time. Likewise, electrophysiology can be used to demonstrate communication between two cells. Non-autonomous effects, such as astrocytes influence can also be studied after iPSC differentiation and co-culturing with more than one cell type. Some differentiation protocols for certain subtypes of neurons already exist, but we still do not know how to differentiate iPSC in culture in all cell types in the human brain. Direct conversion from somatic cells or even from iPSC-derived neural progenitors may be an alternative and can be achieved with a of specific transcription factor cocktail for each cell type. Once obtained, these protocols will enable establishing neuronal circuits in culture, a step forward for understanding diseases and testing new drugs.

Once a consistent cell phenotype is identified in a patient's iPSC-derived neurons, drug testing platforms can be developed with the aim of testing new drugs capable of reversing or alleviate the neuronal defect. After several tests under different experimental conditions, new therapeutic compounds could emerge, benefiting many patients (Figure 1).

\section{The intrinsic variability of pluripotent stem cells}

Available human embryonic stem cells vary considerably in relation to epigenetic markers, gene expression profile and differentiation propensity (Osafune et al., 2008). Apparently, iPSC behave similarly, offering the same intrinsic variability spectrum (Pick et al. 2009). The differences in gene expression profile are generally attributed to the introduction of reprogramming vectors that integrate randomly into the genome, or even to the continued expression of 
pluripotent $\mathrm{cDNAs}$ used in the process. An alternative explanation generated from reprogramming without the use of viral vectors suggests retention of the genetic "memory" of the cell type that it once was (Marchetto et al., 2009). The reason for the variation is still an open question, and more data will be needed for the formulation of hypotheses to be tested. Anyway, this is still a very important point for defining the level of variability among different iPSC clones generated from the same individual. This type of information is essential for the establishment of strict controls during the definition of cell phenotypes.

\section{Conclusions}

Scientists around the world are using iPSC technology to investigate to early stages of human development and to model neurological diseases. So far, this technique has been shown to be restricted to monogenetic diseases, revealing a work methodology for elucidating new molecular pathways responsible for human pathologies. Modeling late-onset diseases such as Alzheimer's, or even psychiatric disorders like autism will require additional factors for the system to be challenged and reveal relevant cellular phenotypes. The potential of cell reprogramming seems to be indeed limited by human creativity and ethical principles defined by society. Neuroscientists in the past could not imagine a scenario in which endless nerve cells of living patients could be constantly generated and studied in laboratories around the world. In turn, researchers in the future will not be able to imagine science without such a tool. Time alone will tell.

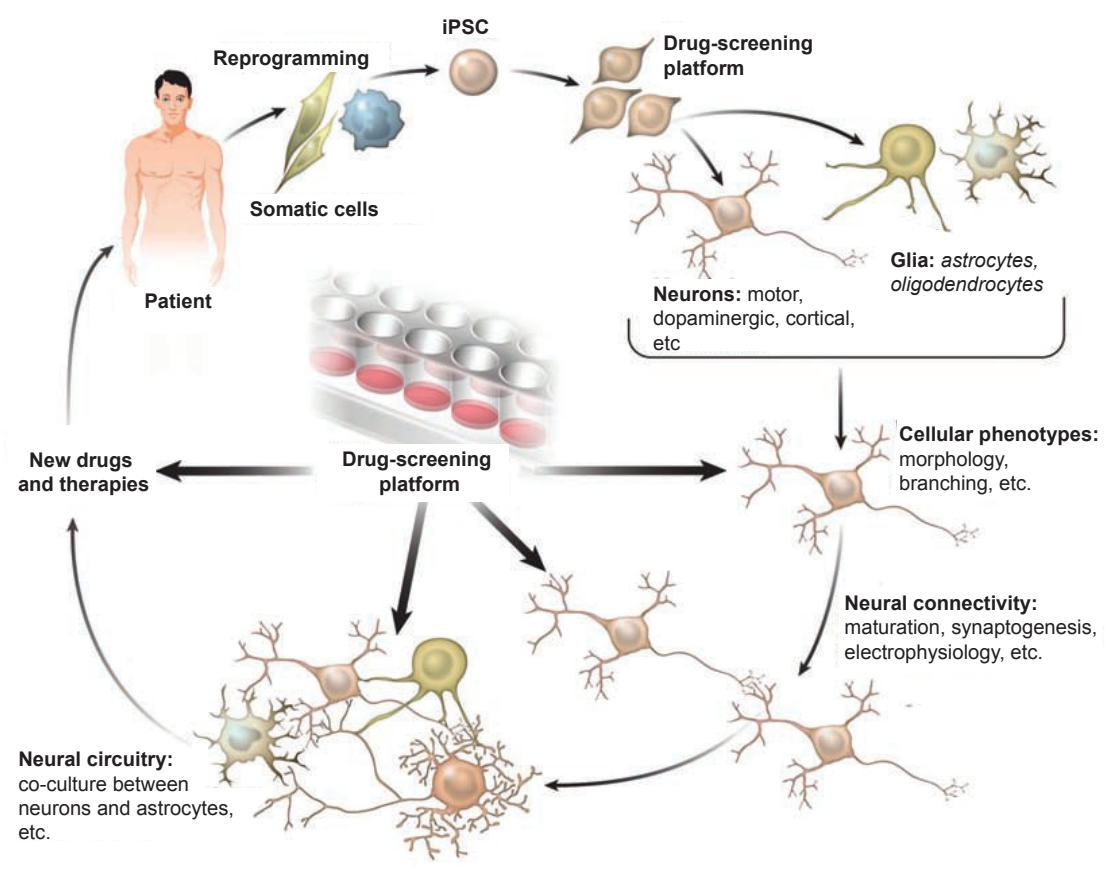

Figure 1 - Modeling of neurological diseases using iPSC technology. Reprogramming of somatic cells of patients and controls, generating isogenic iPSC, i.e., having the same genome as the patient. Neurons can be differentiated into 
subtypes of neurons in accordance with the target cell for each disease. Cellular phenotypes are assessed by measuring neuronal morphology, e.g., soma size, number of processes, etc. Next, connectivity and circuitry integration in culture can be analyzed by different methods well established in neurosciences, such as electrophysiology. In addition, cross talk between neurons and glia can be studied in co-cultures to tease out autonomous events of secondary phenotypes in each type of human disease. Once the phenotype is identified, drug-screening platforms to revert of attenuate the phenotype can be used. New therapies and drugs can emerge from this approach, benefiting numerous neurological patients. Adapted from Marchetto et al. 2010.

\section{References}

BRUNT, P. W.; MCKUSICK, V. A. Familial dysautonomia. A report of genetic and clinical studies, with a review of the literature. Medicine, Baltimore, v.49, p.343-74, 1970.

DIMOS, J. T. et al. Induced pluripotent stem cells generated from patients with ALS can be differentiated into motor neurons. Science, v.321, p.1218-21, 2008.

EBERT, A. D. et al. Induced pluripotent stem cells from a spinal muscular atrophy patient. Nature, v.457, p.277-80, 2009.

HOCKEMEYER, D. et al. Efficient targeting of expressed and silent genes in human ESCs and iPSCs using zinc-finger nucleases. Nat. Biotechnol, v.27, p.851-7, 2009.

LEE, G. et al. Modelling pathogenesis and treatment of familial dysautonomia using patient-specific iPSCs. Nature, v.461, p.402-6, 2009.

LORSON, C. L. et al. SMN oligomerization defect correlates with spinal muscular atrophy severity. Nat. Genet., v.19, p.63-6, 1998.

MARCHETTO, M. C. et al. Transcriptional signature and memory retention of human-induced pluripotent stem cells. PLoS One, v.4, p.e7076, 2009.

Pluripotent stem cells in neurodegenerative and neurodevelopmental diseases. Hum. Mol. Genet., v.19, p.R71-6, 2010.

MUOTRI, A. R. Modeling epilepsy with pluripotent human cells. Epilepsy Behav., v.14, Suppl. 1, p.81-5, 2009.

MUOTRI, A. R.; GAGE, F. H. Generation of neuronal variability and complexity. Nature, v.44l, p.1087-93, 2006.

MUOTRI, A. R. et al. Development of functional human embryonic stem cell-derived neurons in mouse brain. Proc. Natl. Acad. Sci. USA, v.102, p.18644-8, 2005.

OSAFUNE, K. et al. Marked differences in differentiation propensity among human embryonic stem cell lines. Nat. Biotechnol., v.26, p.313-5, 2008.

PARK, I. H. et al. Disease-specific induced pluripotent stem cells. Cell, v.134, p.877$86,2008$.

PICK, M. et al. Clone - and gene - specific aberrations of parental imprinting in human induced pluripotent stem cells. Stem Cells, v.27, p.2686-90, 2009. 
SAMACO, R. C. et al. Epigenetic overlap in autism-spectrum neurodevelopmental disorders:

MECP2 deficiency causes reduced expression of UBE3A and GABRB3. Hum.Mol. Genet., v.14, p.483-92, 2005.

SLAUGENHAUPT, S. A. et al. Tissue-specific expression of a splicing mutation in the IKBKAP gene causes familial dysautonomia. Am. J. Hum. Genet., v.68, p.598-605, 2001.

SOLDNER, F. et al. Parkinson's disease patient-derived induced pluripotent stem cells free of viral reprogramming factors. Cell, v.136, p.964-77, 2009.

SYVERTON, J. T. et al. Studies on the propagation in vitro of poliomyelitis viruses. V. The application of strain HeLa human epithelial cells for isolation and typing. J. Lab.

Clin. Med., v.43, p.286-302, 1954.

TA KAHASHI, K. et al. Induction of pluripotent stem cells from adult human fibroblasts by defined factors. Cell, v.131, p.861-72, 2007.

ZWAKA, T. P.; THOMSON, J. A. Homologous recombination in human embryonic stem cells. Nat. Biotechnol., v.21, p.319-21, 2003.

ABSTRACT - Most of our current knowledge about cellular phenotypes related to neurological diseases was gathered from studies performed in brain tissue collected post-mortem. These samples often represent the end-stage of the disease process and may not represent a fair picture of how the disease developed over time. Furthermore, under these conditions, the pathology may as well be a secondary effect of the disease process or even due to the poor tissue condition and may not represent an authentic cellular phenotype. Likewise, animal models not always recapitulate the pathology from human disorders. In this article, I will present a critical view on the recent advances obtained from disease modeling using human pluripotent stem cells. The focus on cellular reprogramming as a tool to generate patient-specific induced pluripotent stem cells is justified by the great experimental potential, not only for disease modeling, but also as a biotechnological tool for future drug-screening platforms and personalized medicine.

KEYWORDS: Pluripotent stem cells, Cellular reprogramming, Neurological diseases, Drug-screening. 
Alysson Renato Muotri, $\mathrm{PhD}$, is a professor at the University of California, San Diego, Dept. of Pediatrics/Cellular \& Molecular Medicine. @-muotri@ucsd.edu Received on 23 Sept. 2010 and accepted on 29 Sept. 2010. 